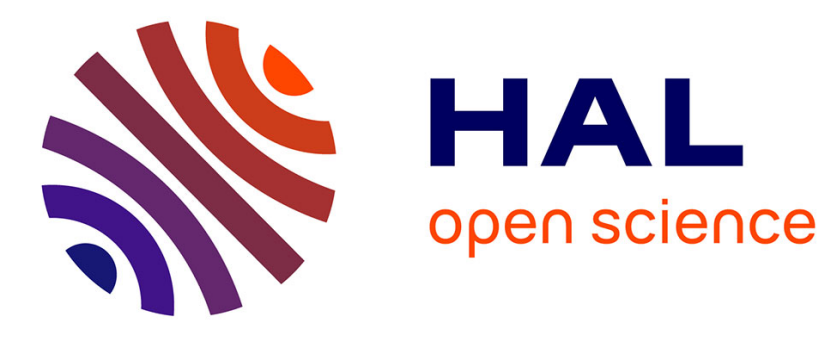

\title{
Chromophore Ordering by Confinement into Carbon Nanotubes
}

\author{
Y Almadori, L Alvarez, R Le Parc, R Aznar, F Fossard, A Loiseau, Bruno \\ Jousselme, S Campidelli, P Hermet, A Belhboub, et al.
}

\section{- To cite this version:}

Y Almadori, L Alvarez, R Le Parc, R Aznar, F Fossard, et al.. Chromophore Ordering by Confinement into Carbon Nanotubes. Journal of Physical Chemistry C, 2014, 118, pp.19462 - 19468. 10.1021/jp505804d . hal-01078525

\section{HAL Id: hal-01078525 \\ https://hal.science/hal-01078525}

Submitted on 29 Oct 2014

HAL is a multi-disciplinary open access archive for the deposit and dissemination of scientific research documents, whether they are published or not. The documents may come from teaching and research institutions in France or abroad, or from public or private research centers.
L'archive ouverte pluridisciplinaire HAL, est destinée au dépôt et à la diffusion de documents scientifiques de niveau recherche, publiés ou non, émanant des établissements d'enseignement et de recherche français ou étrangers, des laboratoires publics ou privés. 


\title{
Chromophore Ordering by Confinement into Carbon Nanotubes
}

\author{
Y. Almadori ${ }^{1,2}$, L. Alvarez ${ }^{* 1,2}$, R. Le Parc ${ }^{1,2}$, R. Aznar ${ }^{1,2}$, F. Fossard ${ }^{3}$, A. Loiseau ${ }^{3}$, B. Jousselme ${ }^{4}$, \\ S. Campidelli ${ }^{4}$, P. Hermet ${ }^{5}$, A. Belhboub ${ }^{1,2,6}$, A. Rahmani ${ }^{6}$ T. Saito $^{7}$, and J-L Bantignies ${ }^{1,2}$ \\ ${ }^{1}$ Laboratoire Charles Coulomb UMR 5521, Université Montpellier 2, F-34095 Montpellier, France \\ ${ }^{2}$ Laboratoire Charles Coulomb UMR 5521, CNRS, F-34095 Montpellier, France \\ ${ }^{3}$ Laboratoire d'étude des microstructures, CNRS-ONERA, 92322 Châtillon, France \\ ${ }^{4}$ CEA-Saclay, IRAMIS, NIMBE, Laboratoire d'Innovation en Chimie des Surfaces et Nanosciences (LICSEN), \\ 91191 Gif-sur-Yvette Cedex, France \\ ${ }^{5}$ Institut Charles Gerhardt Montpellier, UMR 5253 CNRS-UM2-ENSCM-UM1, Université Montpellier 2, Place \\ E. Bataillon, 34095 Montpellier, France \\ ${ }^{6}$ Laboratoire de Physique des matériaux et Modélisation des Systèmes, Université Moulay Ismaïl, Faculté des \\ sciences (Unité associée au CNRST-URAC08), Boîte Postale 11201, Zitoune, 50000 Meknès, Morocco \\ ${ }^{7}$ Nanotube Research Center, National Institute of Advanced Industrial Science and Technology (AIST), Tsukuba \\ 305-8565, Japan
}

\section{Abstract}

We report an experimental study on the confinement of oligothiophene derivatives into single-walled carbon nanotubes over a large range of diameter (from 0.68 to $1.93 \mathrm{~nm}$ ). We evidence by means of Raman spectroscopy and transmission electron microscopy that the supramolecular organizations of the confined oligothiophenes depend on the nanocontainer size. The Raman Radial Breathing Mode frequency is shown to be monitored by both the number of confined molecules into a nanotube section and the competition between oligothiophene/oligothiophene and oligothiophene/tube wall interactions. We finally propose simple Raman criteria to characterize oligothiophene supramolecular organization at the nanoscale.

Keywords: Supramolecular organization, Single wall carbon nanotubes, Dimethyl-quatertiophene, Raman, TEM.

\section{Introduction}

Single-walled carbon nanotubes (NTs) have a one-dimensional hollow space varying from 0.4 to $2.0 \mathrm{~nm}$ and that can encapsulate various molecules. ${ }^{1}$ In these nanohybrid systems, the

* Corresponding author: Laurent.Alvarez@univ-montp2.fr, Phone: +0 33467143 541, Fax: 033467144637 
physical properties of the molecules are supposed to be strongly influenced by their confinement in the one-dimensional cavity and by their specific interactions. ${ }^{2-3}$ For example, members of the fullerene family inserted inside nanotubes give rise to $\pi-\pi$ interactions which are expected to stabilize the guest-host structures. ${ }^{4}$ Calculations have also suggested that confinement of molecules inside NTs can induce structural phase transitions not seen in the bulk phase..$^{5-8}$

Functionalization of nanotubes with organic molecules has attracted significant attention owing to the possibility to modulate NTs properties. ${ }^{9}$ These molecules have been envisaged for optoelectronics ${ }^{10}$ or electron-transport devices ${ }^{11}$ as they display outstanding photophysical properties. Unfortunately, they often suffer from low stability and poor electrical characteristics. ${ }^{10}$ Thus, endohedral functionalization is an efficient way to overcome these drawbacks. One advantage of the encapsulation is that the nanotube protects the internal organic molecules from photo degradation and attacks by external reactive species, thereby preventing undesirable alteration of the organic molecules. ${ }^{12}$ Recently, several experimental works dealt with the encapsulation of oligothiophene derivatives (oTh) into NTs (oTh@NT). ${ }^{13-15}$ Indeed, these systems are particularly interesting as the $\pi$ conjugation of the thiophene molecules provides nonlinear optical properties and intrinsic electrical conductivity. ${ }^{16-20}$

Recent DFT calculations ${ }^{21}$ reported that the electronic states added by the terthiophene molecules inserted into carbon nanotubes would give rise to original optical effects and non radiative relaxation from excited states. Concerning the structure of the nanohybrid material, Yamashita et al. predict that internal thiophene oligomers prefer to be located near the walls of the armchair nanotubes rather than close to the axis. ${ }^{22-23}$ Furthemore, total energy 
calculations suggest that the van der Waals interaction between the oligothiophene and the tube is stronger than the intermolecular interaction. ${ }^{13}$ The different theoretical works suggest a correlation between the structural organization of the guest molecules and the size of the tubes. Nevertheless, up to now, no experimental evidence of such a correlation over a wide range of nanotubes diameter has been reported.

In this work, we investigate experimentally the dependence of the structural properties of NTs filled with methyl-terminated quaterthiophene (4T, figure 1 in supporting information) as a function of the nanotube diameter. We performed a systematic study of hybrid nanotubes in a diameter range from 0.68 to $1.93 \mathrm{~nm}$ using Raman spectroscopy and high resolution transmission microscopy. We found out that $4 \mathrm{~T}$ molecules inside nanotubes form different structures depending on the NT diameters. Different critical diameters are identified, controlled by the interactions between guest thiophene molecules and host nanotube and confined 4T-4T interactions.

\section{EXPERIMENTAL :}

Sample preparation: Four sources of nanotubes have been used in this study: commercial electric arc single-walled carbon nanotubes $(1.2 \mathrm{~nm}<\mathrm{d}<1.6 \mathrm{~nm})$, provided by Carbon Solution $^{24}$ and called NT14 in the following; carbon nanotubes synthesized by the EDiPS $\operatorname{method}^{25}(1.6 \mathrm{~nm}<\mathrm{d}<2 \mathrm{~nm})(\mathrm{NT} 18$ in the following); HiPCO carbon nanotubes $(0.6 \mathrm{~nm}<\mathrm{d}$ $<1.2 \mathrm{~nm}),{ }^{26}$ called NT09 in the following and CoMoCAT carbon nanotube enriched in $(7,6)$ nanotubes $(0.6 \mathrm{~nm}<\mathrm{d}<1 \mathrm{~nm}),{ }^{27}$ NT08 in the following. HiPCO, Carbon Solution and CoMoCAT nanotubes were purified by air oxidation and subsequently treated to remove the catalyst. The eDips nanotubes were purified according to the following protocol: $20 \mathrm{mg}$ of raw SWNT material were sonicated in nitric acid (35 vol. \%) $(150 \mathrm{~mL})$ with a sonic bath (160 $\mathrm{W} \max )(100 \%$ for $5 \mathrm{~min}$ and then $40 \%$ for $15 \mathrm{~min})$ and then heated at $100^{\circ} \mathrm{C}$ for $5 \mathrm{~h}$. The 
suspension was then cooled and vacuum filtered through a PTFE membrane (Sartorius, 0.2 $\mu \mathrm{m})$. While pursuing vacuum filtration, the thick SWNT layer formed on the filtration membrane (buckypaper) was washed by $200 \mathrm{ml}$ of deionized water; $\mathrm{pH}$ was monitored during the washing and is about 7 at the end of the process. The nanotubes were redispersed in $\mathrm{NaOH} 1 \mathrm{M}(100 \mathrm{~mL})$ using the sonic bath $(100 \%$ for $10 \mathrm{~min})$ and then filtered through a PTFE membrane and washed with $\mathrm{NaOH} 1 \mathrm{M}$, deionized water and then $\mathrm{HCl} 1 \mathrm{M}$ followed by deionized water until the filtrate was neutral. Finally, the bucky paper was redispersed in hydrogen peroxide $(30 \%)(150 \mathrm{~mL})$ using the sonic bath $(100 \%$ for $5 \mathrm{~min}$ and then $40 \%$ for $10 \mathrm{~min}$ ). The suspension was heated at $100^{\circ} \mathrm{C}$ for $1 \mathrm{~h}$, cooled down at room temperature and then vacuum filtered through a PTFE membrane. The nanotubes were washed by $200 \mathrm{ml}$ of deionized water and then dried at $50^{\circ} \mathrm{C}$ under high vacuum. The overall yield of the purification process was $60 \%(12 \mathrm{mg})$.

Encapsulation of $4 \mathrm{~T}$ into carbon nanotubes is performed using the vapour reaction method previously described. ${ }^{15}$ Before the encapsulation treatment, carbon nanotubes are outgased at $300^{\circ} \mathrm{C}$ for 48 hours. Then, NTs are mixed with $4 \mathrm{~T}-\mathrm{CH} 3$ in weight ratio: $\mathrm{w}_{\mathrm{NTS}} / \mathrm{w}_{4 \mathrm{~T}-\mathrm{CH} 3}=0.5$, in glove box and outgased under $2.10^{-6}$ mbar at ambient temperature for 1 hour. Then, NTs with $4 \mathrm{~T}$ are sealed in glass tube at $2.10^{-6} \mathrm{mbar}$ and heated to $250^{\circ} \mathrm{C}$ for 72 hours. Sublimation step is then performed. The sample was then washed with organic solvent and stored in the oven at $120^{\circ} \mathrm{C}$ for 24 hours. The hybrid material is named 4T@NT in the following.

Raman spectroscopy: Micro Raman experiments have been performed on a triple monochromator spectrometer (Jobin Yvon T64000), equipped with a charge-coupled detector, in a back scattering geometry, using the fixed 457.9, 488.0, 514.5, 568.2 and $647.1 \mathrm{~nm}$ excitation wavelengths and a Ti-Sapphire tunable source in the near infrared region. The spot size is around few microns in diameter probing an ensemble of nanotubes. 
In order to prevent the heating of the tubes and oligomers, the laser power was adjusted at $100 \mu \mathrm{W}$ with a spot diameter of about $3 \mu \mathrm{m}$ using a $50 \mathrm{X}$ objective. The resolution is about 2 $\mathrm{cm}^{-1}$. At least four spatially separated area of each sample were probe to ensure homogeneity.

HR-TEM: The TEM samples were prepared by dispersing the NTs powders in ethanol. The dispersions were ultrasonicated and subsequently deposited on a holey carbon $3 \mathrm{~mm}$ copper grids. TEM images were made using two TEMs: a ZEISS Libra $200 \mathrm{MC}$ operating at $200 \mathrm{kV}$ was used to study different sources of nanotubes. A Cs-corrected JEOL ARM200 CC operating at $80 \mathrm{kV}$ was used to quantify precisely the encapsulation.

\section{Computational details:}

DFT calculations were performed with the SIESTA package ${ }^{28}$ on a 4T@ $(11,0)$. In this model, we use a $(11,0)$ nanotube of $29.82 \AA$ length (diameter of $8.6 \AA$ ) with periodic conditions along its axis. The vacuum size was of $11 \AA$ in the two other directions to avoid interactions between adjacent tubes. The guest molecule is a quaterthiophene oligomer bounded with methyl groups (4T). The first Brillouin zone was sampled using $8 \boldsymbol{k}$-points along the nanotube axis. The atomic positions were relaxed at fixed lattice parameters using a conjugate gradient until the maximum residual force on the atoms was smaller than $0.02 \mathrm{eV} / \AA$. Exchangecorrelation effects were handled within the generalized gradient approximation (GGA) as proposed by Perdew, Burke and Ernzerhof. ${ }^{29}$ Core electrons are replaced by nonlocal normconserving pseudopotentials. ${ }^{30}$ The valence electrons were described by localized pseudoatomic orbitals with a double- $\zeta$ singly polarized (DZP) basis set. ${ }^{31}$ The cutoff radii for the $s$ and $p$ orbitals were respectively 4.99 and 6.25 a.u. for the carbon atoms, and 4.97 and 
6.22 a.u. for sulfur atoms, whereas it was 6.05 a.u. for the $s$ orbital of hydrogen atoms. A real space integration was performed on a regular grid corresponding to a plane-wave cutoff around 300 Ry. We also use van der Waals corrections (DFT-D) between the tube and the guest molecule according to the Grimme approach. ${ }^{32}$ This approach consists in adding a semiempirical dispersion potential (a simple pair-wise force field) to the conventional Kohn-Sham DFT energy.

\section{RESULTS AND DISCUSSION :}

Raman spectra of carbon nanotubes are very sensitive to charge transfers and/or environmental effects, ${ }^{33-34}$ making thus possible to investigate very efficiently the interaction between oligothiophene molecules and nanotubes. The G-band undergoes significant modifications after 4T encapsulation associated to a charge transfer, already discussed in reference 15

Here, we will focus on the low frequency region located between 80 and $350 \mathrm{~cm}^{-1}$ where the Radial Breathing Modes (RBM) related to the tube diameters are observed. The relationship between the frequency $\omega$ and the tube diameter $d$ is usually given by the following equation: $\omega\left(\mathrm{cm}^{-1}\right)=A / d(\mathrm{~nm})+B$ where $A$ and $B$ are two constants. ${ }^{33-43}$ The following relationship $d=\frac{217,8}{\omega_{R B M}-15,7}$ from the reference ${ }^{40}$ is used in this work to determine the carbon nanotube diameters.

Figure 1 displays the Raman spectra in the RBM frequency range at different laser excitation wavelengths for empty nanotubes (open circles) and 4T@NT samples (full squares). The diameter distribution is strongly dependent on the nanotube source. The modes clearly exhibit a frequency up-shift after encapsulation for different laser excitation energies. The relative upshifts $\left(\Delta \omega_{\mathrm{RBM}}=\omega_{\mathrm{RBM}}(4 \mathrm{~T} @ \mathrm{NT})-\omega_{\mathrm{RBM}}(\mathrm{NT})\right)$ for all the nanotubes investigated are plotted on 
figure 2 as a function of the NT diameter and the different excitation wavelengths. The behavior is clearly non-monotonic and displays a complex dependence with the nanotube diameter over the whole range.

For small diameter NT, typically between 0.68 and $1.1 \mathrm{~nm}$, the relative up-shifts are almost constant. This trend is well characterized by a plateau around a value of $2 \mathrm{~cm}^{-1}$ for $\Delta \omega_{R B M}$. A linear behavior is observed for diameters between 1.1 and $1.4 \mathrm{~nm}$. Another plateau can be also considered between 1.4 and $1.65 \mathrm{~nm}$, suggesting that the RBM shits are independent of the NT diameter in this range. To confirm this assumption, a detailed study of the RBM frequency shifts in this diameter range for the encapsulation into NT14 carbon nanotubes of other kinds of oligothiophenes (quaterthiophene $(\alpha-4 T)$, sexithiophene $(\alpha-6 \mathrm{~T})$ and dimethylquaterthiophene (4T)) is displayed on the inset of figure 2. Those data indicate that the relative RBM up-shift upon encapsulation is rather constant around a value of $7.5 \mathrm{~cm}^{-1}$ in this diameter range whatever the nature of the confined molecules. Finally, for diameter larger than $1.6 \mathrm{~nm}$ the behavior is shown to be linear despite the low number of experimental points.

The RBM behaviors can be associated to the amount of molecules confined into a nanotube section and their supramolecular organization as suggested by experimental work on water encapsulation $^{44}$ and theoretical studies on sexithiophene into nanotubes. ${ }^{13}$

To study the structural organization of the nanohybrid systems, we performed HRTEM measurements on 4T@NT. Figure 3 displays some representative HRTEM micrographs of carbon nanotubes with different diameters filled with 4T (left) and their corresponding contrast profiles (right). Concerning the smallest carbon nanotube, we can distinguish three minima in the contrast profile of figure 3.d. The minima on the sides correspond to the carbon nanotube walls. The minimum in between is reasonably associated to a $4 \mathrm{~T}$ molecule confined inside the carbon nanotube. The observed configuration is one isolated chain inserted in the 
carbon nanotube and centered along the nanotube main axis. For the nanotube with a diameter of $1.4 \mathrm{~nm}$, two minima are located in between the NT walls. Thus, the configuration in this case consists in two molecular chains inserted in the same nanotube section.

For the largest carbon nanotube (figure 3c), one can observe three mimima in between the nanotube walls (figure 3f), showing that a third chain is inserted. Thus, to explain the RBM shifts as a function of the nanotube diameter, we propose the model described below. For diameter between 0.68 and $1.1 \mathrm{~nm}$, only one single molecule fit in the tube. Indeed, the oligothiophene width is around $0.48 \mathrm{~nm}$ for an isolated molecule in its most stable structure, when adjacent rings are non-parallel to one another thanks to a rotation around the connecting bond. Furthermore, the van der Walls distance to the tube wall is roughly $0.32 \mathrm{~nm},{ }^{13}$ so that a $1.1 \mathrm{~nm}$ diameter tube can accommodate only one molecule $(0.48+2 \times 0.32=1.12 \mathrm{~nm})$. Below $1.1 \mathrm{~nm}$, we assume that the oligothiophene size is strongly reduced under nano confinement by aligning all the thiophene rings to form a nearly planar structure. This hypothesis is supported by our calculations. Indeed, after relaxation, the $4 \mathrm{~T}$ molecule inside a $(11,0)$ nanotube displays dihedral angles between thiophene rings smaller than $1.1^{\circ}$ and its distance to the nanotubes wall is $4 \AA$ (figure 4.a). Therefore, a planar molecule can incorporate nanotubes with diameter down to $0.68 \mathrm{~nm}$, as experimentally observed. Our calculations also predict that $4 \mathrm{~T}$ molecules cannot enter a $(8,0)$ nanotube (diameter $\mathrm{d}=0.63 \mathrm{~nm}$ ) as no stable configuration is found out. Therefore, the critical NT diameter for 4T encapsulation is around $0.65 \mathrm{~nm}$.

For nanotubes diameter between 1.10 and $1.45 \mathrm{~nm}$, a second chain is inserted (figure 4.b). DFT calculations for $4 \mathrm{~T}$ confined into a $(17,0)$ nanotube $($ diameter $=1.35 \AA$ ) give the following structural parameters: the distance between the two molecules is $6,5 \AA$ whereas the distance between the molecules and the tube wall is $3.4 \AA$, close to the inter-sheet distance in graphite (figure 4.b). 
Around $1.1 \mathrm{~nm}$, the molecules are very close to each other and strongly interact, leading to a slight upshift. When the NT diameter increases, the molecules get further and further away, interacting more and more with the NT wall, giving rise to the linear behavior observed on figure 2. This assumption is consistent with theoretical works since the molecules are calculated to be more and more distant as the nanotube diameter increases as a consequence of a higher affinity of the molecule with the nanotube walls. ${ }^{10,13,45-48}$ Between 1.40 and 1.65 $\mathrm{nm}$, the NT diameter is large enough to accommodate only two molecules close to the wall. Beyond $1.65 \mathrm{~nm}$, the insertion of a third molecule is possible and the distance to the nanotube wall decreases when the tube diameter increases.

The RBM shifts induced by non-covalent endohedral functionalization of nanotubes with different species can have different origins such as dispersive interactions, charge transfer, mechanical strain... For fullerene molecules $\left(\mathrm{C}_{60}, \mathrm{C}_{70}, \mathrm{C}_{76} \ldots\right)$, the dispersive interactions (van der Waals), generally lead to a small down-shift $\left(2\right.$ or $\left.3 \mathrm{~cm}^{-1}\right)$ according to the reference Britz and Khlobystov ${ }^{49}$ and references therein. However, the shift direction could depend on the nanotube diameter as reported by S. Okada ${ }^{50}$ and S. Joung et al. ${ }^{51}$ Indeed, encapsulation into large diameter tubes (above $1.37 \mathrm{~nm}$ ) leads to a down-shift due to an electronic hybridization between NT and $\mathrm{C}_{60}$ whereas insertion into smaller diameter tubes leads to a small up-shift caused by a steric hindrance. Charge transfer (positive or negative) onto individual metallic nanotubes has been shown to induce a small RBM up-shift of about $2 \mathrm{~cm}^{-1}$ due to a renormalization effect as observed for the Raman G-band. ${ }^{52}$ This behavior is observed when electron acceptor molecules are encapsulated into bundles of semiconducting or metallic tubes $^{49}$. By contrast, insertion of electron donor species such as alkali metals gives rise to a small down-shift for lithium, ${ }^{53}$ no shift for rubidium ${ }^{54}$ and a slight up-shift for cesium. ${ }^{55}$ As the $4 \mathrm{~T}$ molecule is an electron donor, the physical interactions with the nanotubes are 
probably dispersive and combined to a slight charge transfer (as evidenced in ref.15). It is worth mentioning that the RBM shifts are at most $-3 \mathrm{~cm}^{-1}$ for dispersive interactions ${ }^{49-51}$ and $+2 \mathrm{~cm}^{-1}$ for charge transfer. ${ }^{52,55}$ Thus, for $4 \mathrm{~T}$ confined into NT, one can reasonably expect RBM shifts in this wavenumber range or close to zero if both interactions cancel each other. However, figure 2 displays RBM shifts that reach $+8 \mathrm{~cm}^{-1}$ at the second plateau and up to +17 $\mathrm{cm}^{-1}$ for the largest diameter tube. Then, the behavior of the RBM shifts over the large diameter range cannot be simply understood as a superposition of dispersive interactions and charge transfer. H. Kataura et al. interpreted the RBM behavior of nanotubes (shifts of about $+5 \mathrm{~cm}^{-1}$ and intensity reduction) after Zn-diphenylporphyrin encapsulation (good electron donor $\left.^{49}\right)$ as a nanotube deformation due to the large size of the molecule $(1 \mathrm{~nm}) .{ }^{56} \mathrm{We}$ therefore believe that the $4 \mathrm{~T}$ molecules induce a mechanical strain on the nanotubes whose energy depends on the amount of molecules confined and on the closeness between the $4 \mathrm{~T}$ and the NT. This closeness depends on the NT diameter as suggested by our DFT calculations.

Thus, according to our Raman investigations, the magnitude of the RBM upshifts as a function of the nanotube diameter is governed by two different parameters: the number of confined molecules lying in a nanotube section and the physical interaction taking place either between the molecules themselves or between the molecules and the nanotubes walls.

\section{Conclusion}

In summary, combined Raman and HRTEM measurements allow to determine the supramolecular organization of 4T confined into single-walled carbon nanotubes. We evidence different critical nanotubes diameters. Below $1.1 \mathrm{~nm}$, only one molecule is encapsulated. Between 1.1 and $1.6 \mathrm{~nm}$, two oligothiophenes are confined. Above $1.6 \mathrm{~nm}$, three molecules are inserted. The Raman Radial Breathing Mode frequency displays a 
nonmonotonic behavior with the NT diameter. It turns out to be monitored by both the number of confined molecules into a nanotube section and the competition between oligothiophene/oligothiophene and oligothiophene/tube walls interactions. Our results allow establishing simple Raman criteria to characterize oligothiophenes supramolecular organization at the nanoscale by investigating carefully the magnitude of the RBM up-shifts. To go further in our investigations, nanotubes with specific diameters are required. For instance, diameters around $1.3 \mathrm{~nm}$ would allow obtaining more experimental points in the linear regime. Diameters larger than $2 \mathrm{~nm}$ would allow reaching the third plateau which should correspond to three molecular chains interacting with the nanotubes walls without any interaction between the molecules themselves. 


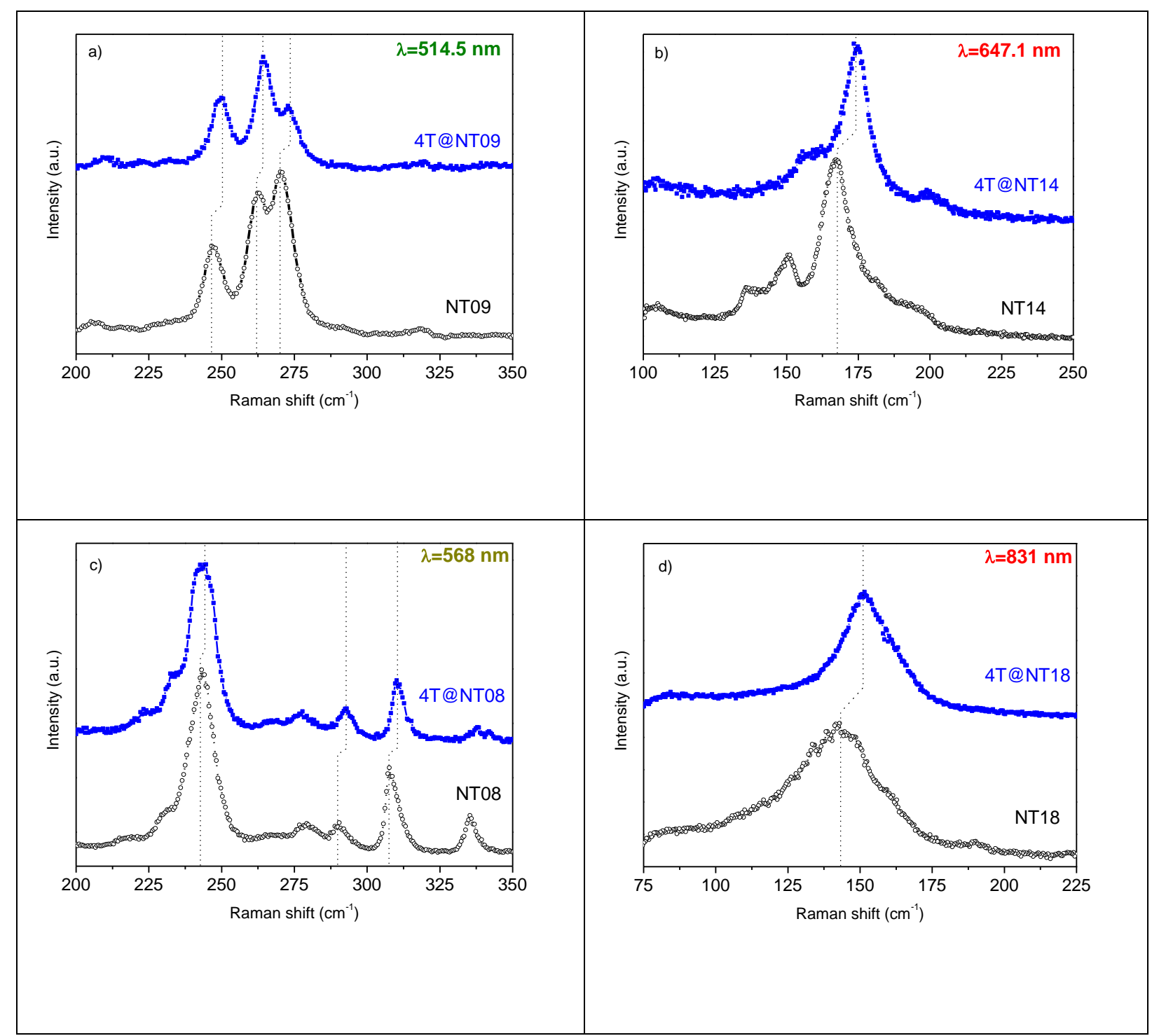

Figure 1: Raman spectra of NT and 4T@NT in the RBM range recorded at different laser excitation wavelengths 


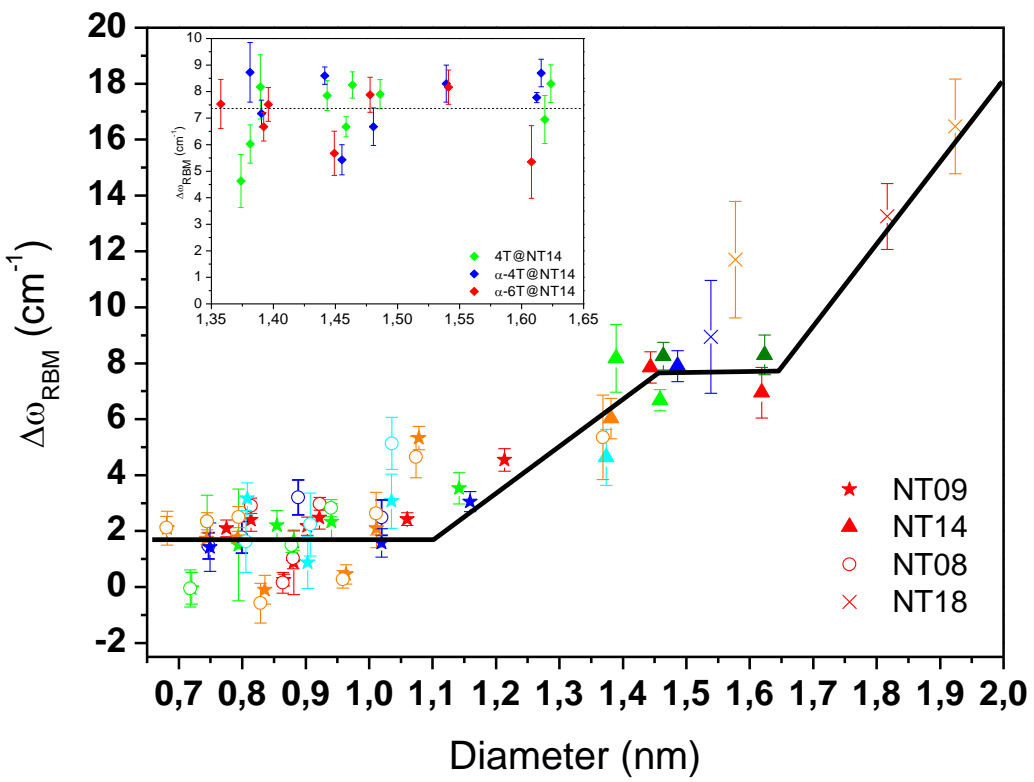

Figure 2: RBM relative up-shifts after encapsulation of 4T molecules inside NT08 (open circles), NT09 (stars), NT14 (triangles) and NT18 (crosses) carbon nanotubes. The colors of the symbols correspond to the laser excitation wavelengths (cyan $=458 \mathrm{~nm}$, blue $=488 \mathrm{~nm}$, dark green=532 nm, green=514.5 nm, red=647.1 nm, orange $=568 \mathrm{~nm}$ ). The black straight lines are guide for eyes. Inset: RBM relative up-shifts after encapsulation of oligothiophene molecules (4T, $\alpha-4 T$ and $\alpha-6 T)$ inside NT14 carbon nanotubes. 


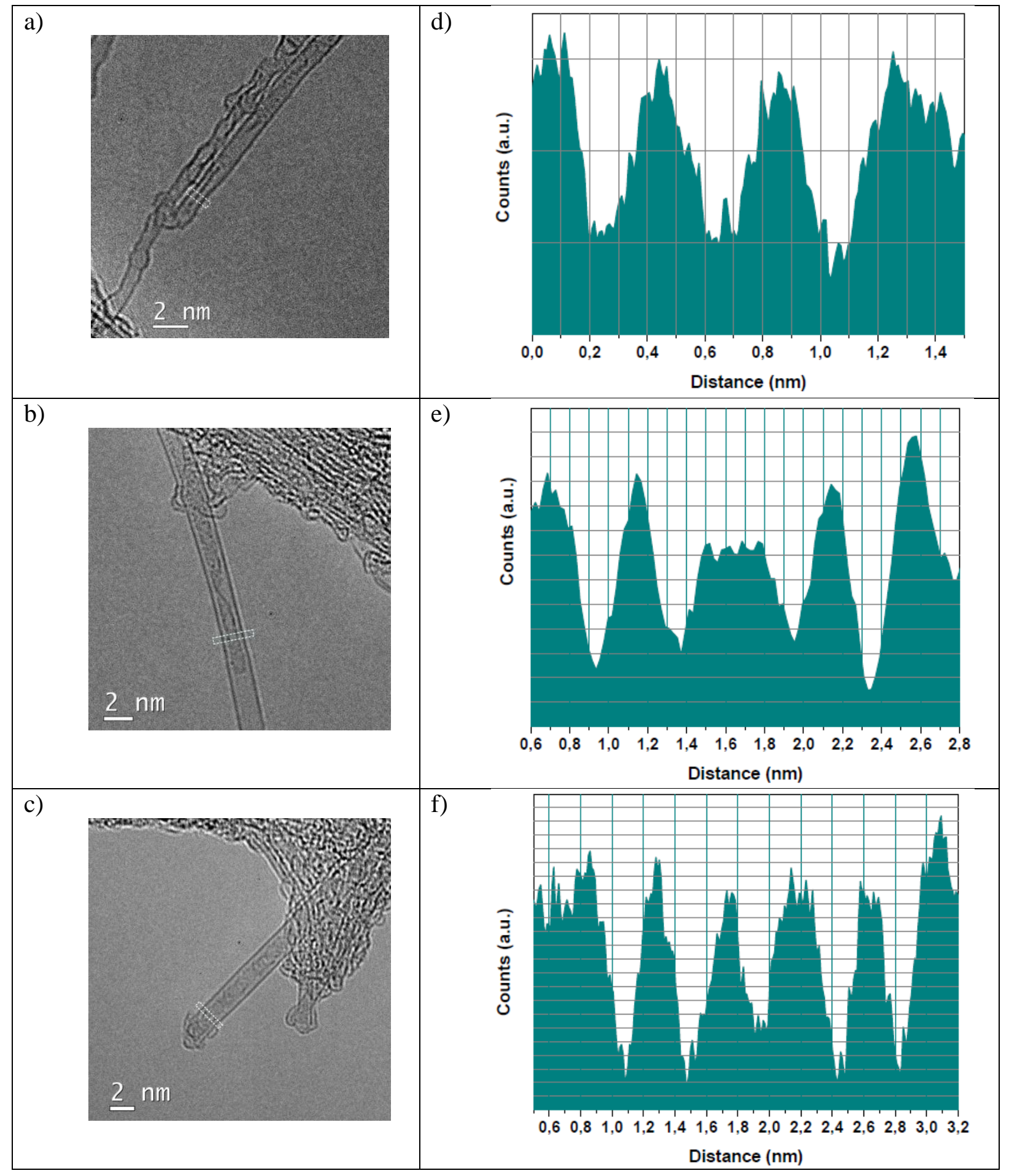

Figure 3: $(a, b, c)$ HRTEM micrograph of carbon nanotubes with different diameters filled with $4 T$ molecules. $(d, e, f)$ Contrast profiles (along the rectangle) of the left carbon nanotubes 


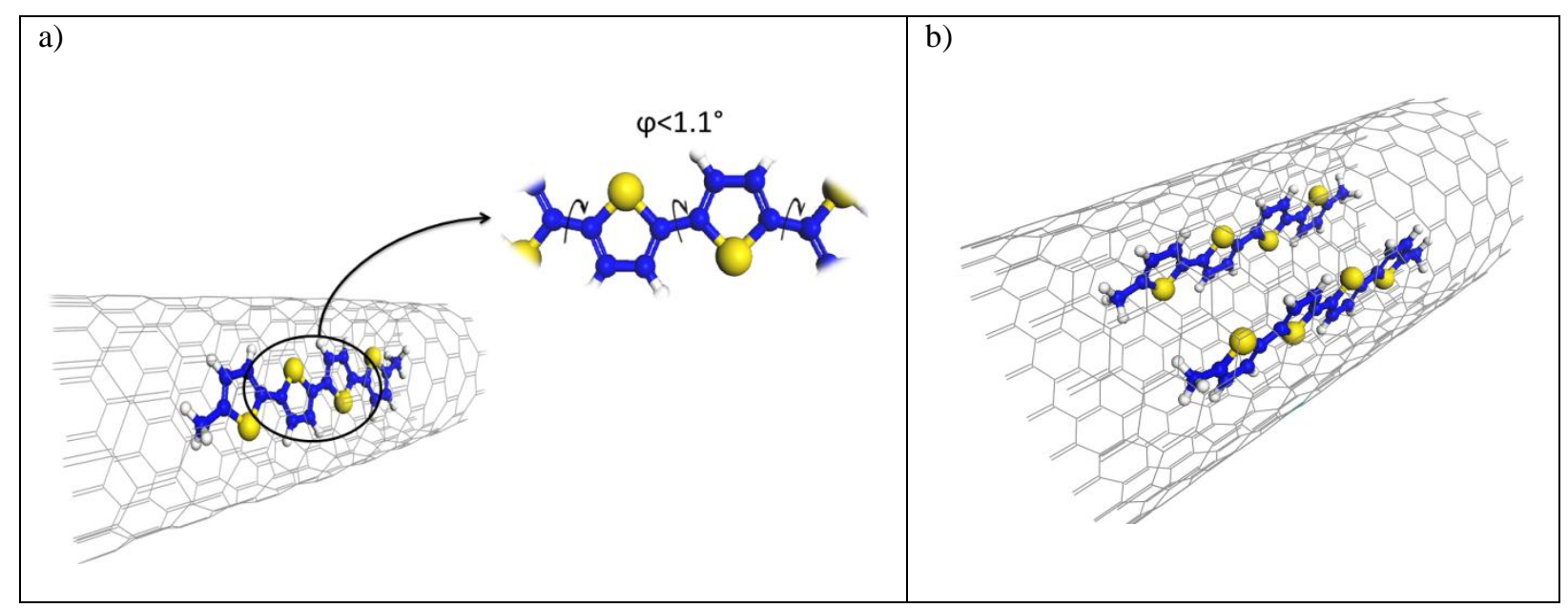

Figure 4: Optimized 4T@NT structures for a $(11,0)$ tube $(\mathrm{a})$ and a $(17,0)$ tube (b)

\section{Acknowledgment :}

Authors wish to thank the METSA research foundation for giving access to the Cs-corrected TEM of $\mathrm{MPQ}$-Paris Diderot laboratory.

\section{Supporting Information available:}

This information is available free of charge via the Internet at http://pubs.acs.org

\section{Bibliographie}

(1) Khlobystov, A. N.; Britz, D. A.; Briggs, G. A. D. Molecules in Carbon Nanotubes. Acc. Chem. Res. 2005, 38, 901-909.

(2) Hornbaker, D. J.; Kahng, S. J.; Misra, S.; Smith, B. W.; Johnson, A. T.; Mele, E. J.; Luzzi, D. E.; Yazdani, A. Mapping the One-Dimensional Electronic States of Nanotube Peapod Structures. Science 2002, 295, 828-831.

(3) Campestrini, S.; Corvaja, C.; De Nardi, M.; Ducati, C.; Franco, L.; Maggini, M.; Meneghetti, M.; Menna, E.; Ruaro, G. Investigation of the Inner Environment of Carbon Nanotubes with a FullereneNitroxide Probe. Small 2008, 4, 350-356.

(4) Smith, B. W.; Monthioux, M.; Luzzi, D. E. Encapsulated $C_{60}$ in Carbon Nanotubes. Nature 1998, 396, 323-324.

(5) Hummer, G.; Rasaiah, J. C.; Noworyta, J. P. Water Conduction through the Hydrophobic Channel of a Carbon Nanotube. Nature 2001, 414, 188-190.

(6) Kim, G.; Kim, Y.; Ihm, J. Encapsulation and Polymerization of Acetylene Molecules inside a Carbon Nanotube. Chem. Phys. Lett. 2005, 415, 279-282.

(7) Guan, L.; Suenaga, K.; Shi, Z.; Gu, Z.; lijima, S. Polymorphic Structures of lodine and Their Phase Transition in Confined Nanospace. Nano Lett. 2007, 7, 1532-1535.

(8) Chorro, M.; Kane, G.; Alvarez, L.; Cambedouzou, J.; Paineau, E.; Rossberg, A.; Kociak, M.; Aznar, R.; Pascarelli, S.; Launois, P. et al. 1D-Confinement of Polyiodides inside Single-Wall Carbon Nanotubes. Carbon 2013, 52, 100-108. 
(9) Balasubramanian, K.; Burghard, M. Chemically Functionalized Carbon Nanotubes. Small 2005, 1, 180-192.

(10) Yanagi, K.; lakoubovskii, K.; Kazaoui, S.; Minami, N.; Maniwa, Y.; Miyata, Y.; Kataura, H. LightHarvesting Function of Beta-Carotene inside Carbon Nanotubes. Phys. Rev. B 2006, 74, 155420.

(11) Shimada, T.; Okazaki, T.; Taniguchi, R.; Sugai, T.; Shinohara, H.; Suenaga, K.; Ohno, Y.; Mizuno, S.; Kishimoto, S.; Mizutani, T. Ambipolar Field-Effect Transistor Behavior of Gd@C-82

Metallofullerene Peapods. Appl. Phys. Lett. 2002, 81, 4067-4069.

(12) Yanagi, K.; Miyata, Y.; Kataura, H. Highly Stabilized Beta-Carotene in Carbon Nanotubes. Adv. Mater. 2006, 18, 437-441.

(13) Loi, M. A.; Gao, J.; Cordella, F.; Blondeau, P.; Menna, E.; Bartova, B.; Hebert, C.; Lazar, S.; Botton, G. A.; Milko, M. et al. Encapsulation of Conjugated Oligomers in Single-Walled Carbon Nanotubes: Towards Nanohybrids for Photonic Devices. Adv. Mater. 2010, 22, 1635-1639.

(14) Kalbac, M.; Kavan, L.; Gorantla, S.; Gemming, T.; Dunsch, L. Sexithiophene Encapsulated in a Single-Walled Carbon Nanotube: An in Situ Raman Spectroelectrochemical Study of a Peapod Structure. Chem.-Eur. J. 2010, 16, 11753-11759.

(15) Alvarez, L.; Almadori, Y.; Arenal, R.; Babaa, R.; Michel, T.; Le Parc, R.; Bantignies, J. L.; Jousselme, B.; Palacin, S.; Hermet, P. et al. Charge Transfer Evidence between Carbon Nanotubes and Encapsulated Conjugated Oligomers. J. Phys. Chem. C 2011, 115, 11898-11905.

(16) Brédas, J. L.; Street, G. B. Polarons, Bipolarons, and Solitons in Conducting Polymers. Acc. Chem. Res. 1985, 18, 309-315.

(17) Swager, T. M. The Molecular Wire Approach to Sensory Signal Amplification. Acc. Chem. Res. 1998, 31, 201-207.

(18) McQuade, D. T.; Pullen, A. E.; Swager, T. M. Conjugated Polymer-Based Chemical Sensors. Chem. Rev. 2000, 100, 2537-2574.

(19) Kertesz, M.; Choi, C. H.; Yang, S. J. Conjugated Polymers and Aromaticity. Chem. Rev. 2005, 105, 3448-3481.

(20) Chen, Z. F.; Wannere, C. S.; Corminboeuf, C.; Puchta, R.; Schleyer, P. V. Nucleus-Independent Chemical Shifts as an Aromaticity Criterion. Chem. Rev. 2005, 105, 3842-3888.

(21) Orellana, W.; Vasquez, S. O. Endohedral Terthiophene in Zigzag Carbon Nanotubes: Density Functional Calculations. Phys. Rev. B 2006, 74, 125419.

(22) Yamashita, H.; Yumura, T. The Role of Weak Bonding in Determining the Structure of Thiophene Oligomers inside Carbon Nanotubes. J. Phys. Chem. C 2012, 116, 9681-9690.

(23) Yumura, T.; Yamashita, H. Modulating the Electronic Properties of Multimeric Thiophene Oligomers by Utilizing Carbon Nanotube Confinement. J. Phys. Chem. C 2014, 118, 5510-5522.

(24) Carbon Solution Inc, http://www.carbonsolution.com.

(25) Saito, T.; Ohshima, S.; Okazaki, T.; Ohmori, S.; Yumura, M.; lijima, S. Selective Diameter Control of Single-Walled Carbon Nanotubes in the Gas-Phase Synthesis. J. Nanosci. Nanotechnol. 2008, 8, 6153-6157.

(26) Nikolaev, P.; Bronikowski, M. J.; Bradley, R. K.; Rohmund, F.; Colbert, D. T.; Smith, K. A.; Smalley, R. E. Gas-Phase Catalytic Growth of Single-Walled Carbon Nanotubes from Carbon Monoxide. Chem. Phys. Lett. 1999, 313, 91-97.

(27) http://www.sigmaaldrich.com.

(28) Sanchez-Portal, D.; Ordejon, P.; Artacho, E.; Soler, J. M. Density-Functional Method for Very Large Systems with LCAO Basis Sets. Int. J. Quantum Chem. 1997, 65, 453-461.

(29) Perdew, J. P.; Burke, K.; Ernzerhof, M. Generalized Gradient Approximation Made Simple. Phys. Rev. Lett. 1996, 77, 3865-3868.

(30) Troullier, N.; Martins, J. L. Efficient Pseudopotentials for Plane-Wave Calculations. Phys. Rev. B 1991, 43, 1993-2006.

(31) Artacho, E.; Sanchez-Portal, D.; Ordejon, P.; Garcia, A.; Soler, J. M. Linear-Scaling Ab-Initio Calculations for Large and Complex Systems. Phys. Status Solidi B 1999, 215, 809-817.

(32) Grimme, S. Semiempirical GGA-Type Density Functional Constructed with a Long-Range Dispersion Correction. J. Comput. Chem. 2006, 27, 1787-1799. 
(33) Dresselhaus, M. S.; Dresselhaus, G.; Saito, R.; Jorio, A. Raman Spectroscopy of Carbon Nanotubes. Phys. Rep.-Rev. Sec. Phys. Lett. 2005, 409, 47-99.

(34) Thomsen, C.; Reich, S.; Maultzsch, J. Resonant Raman Spectroscopy of Nanotubes. Philos. Trans. R. Soc. Lond. Ser. A-Math. Phys. Eng. Sci. 2004, 362, 2337-2359.

(35) Araujo, P. T.; Pesce, P. B. C.; Dresselhaus, M. S.; Sato, K.; Saito, R.; Jorio, A. Resonance Raman Spectroscopy of the Radial Breathing Modes in Carbon Nanotubes. Physica E 2010, 42, 1251-1261.

(36) Machon, M.; Reich, S.; Telg, H.; Maultzsch, J.; Ordejon, P.; Thomsen, C. Strength of Radial Breathing Mode in Single-Walled Carbon Nanotubes. Phys. Rev. B 2005, 71, 035416.

(37) Strano, M. S.; Doorn, S. K.; Haroz, E. H.; Kittrell, C.; Hauge, R. H.; Smalley, R. E. Assignment of (N, M) Raman and Optical Features of Metallic Single-Walled Carbon Nanotubes. Nano Lett. 2003, 3, 1091-1096.

(38) Telg, H.; Maultzsch, J.; Reich, S.; Hennrich, F.; Thomsen, C. Chirality Distribution and Transition Energies of Carbon Nanotubes. Phys. Rev. Lett. 2004, 93, 177401.

(39) Jorio, A.; Fantini, C.; Pimenta, M. A.; Capaz, R. B.; Samsonidze, G. G.; Dresselhaus, G.; Dresselhaus, M. S.; Jiang, J.; Kobayashi, N.; Gruneis, A. et al. Resonance Raman Spectroscopy (N,M)Dependent Effects in Small-Diameter Single-Wall Carbon Nanotubes. Phys. Rev. B 2005, 71, 075401. (40) Araujo, P. T.; Doorn, S. K.; Kilina, S.; Tretiak, S.; Einarsson, E.; Maruyama, S.; Chacham, H.; Pimenta, M. A.; Jorio, A. Third and Fourth Optical Transitions in Semiconducting Carbon Nanotubes. Phys. Rev. Lett. 2007, 98, 067401.

(41) Sauvajol, J. L.; Anglaret, E.; Rols, S.; Alvarez, L. Phonons in Single Wall Carbon Nanotube Bundles. Carbon 2002, 40, 1697-1714.

(42) Rao, A. M.; Chen, J.; Richter, E.; Schlecht, U.; Eklund, P. C.; Haddon, R. C.; Venkateswaran, U. D.; Kwon, Y. K.; Tomanek, D. Effect of Van Der Waals Interactions on the Raman Modes in Single Walled Carbon Nanotubes. Phys. Rev. Lett. 2001, 86, 3895-3898.

(43) Alvarez, L.; Righi, A.; Rols, S.; Anglaret, E.; Sauvajol, J. L. Excitation Energy Dependence of the Raman Spectrum of Single-Walled Carbon Nanotubes. Chem. Phys. Lett. 2000, 320, 441-447.

(44) Cambré, S.; Schoeters, B.; Luyckx, S.; Goovaerts, E.; Wenseleers, W. Experimental Observation of Single-File Water Filling of Thin Single-Wall Carbon Nanotubes Down to Chiral Index (5,3). Phys. Rev. Lett. 2010, 104, 207401.

(45) Yanagi, K.; lakoubovskii, K.; Matsui, H.; Matsuzaki, H.; Okamoto, H.; Miyata, Y.; Maniwa, Y.; Kazaoui, S.; Minami, N.; Kataura, H. Photosensitive Function of Encapsulated Dye in Carbon Nanotubes. J. Am. Chem. Soc. 2007, 129, 4992-4997.

(46) Gao, J.; Blondeau, P.; Salice, P.; Menna, E.; Bartova, B.; Hebert, C.; Leschner, J.; Kaiser, U.; Milko, M.; Ambrosch-Draxl, C. et al. Electronic Interactions between "Pea" and "Pod": The Case of Oligothiophenes Encapsulated in Carbon Nanotubes. Small 2011, 7, 1807-1815.

(47) Kuwahara, R.; Kudo, Y.; Morisato, T.; Ohno, K. Encapsulation of Carbon Chain Molecules; in Single-Walled Carbon Nanotubes. J. Phys. Chem. A 2011, 115, 5147-5156.

(48) McIntosh, G. C.; Tománek, D.; Park, Y. W. Energetics and Electronic Structure of a Polyacetylene Chain Contained in a Carbon Nanotube. Phys. Rev. B 2003, 67, 125419.

(49) Britz, D. A.; Khlobystov, A. N. Noncovalent Interactions of Molecules with Single Walled Carbon Nanotubes. Chem. Soc. Rev. 2006, 35, 637-659.

(50) Okada, S. Radial-Breathing Mode Frequencies for Nanotubes Encapsulating Fullerenes. Chem. Phys. Lett. 2007, 438, 59-62.

(51) Joung, S.-K.; Okazaki, T.; Kishi, N.; Okada, S.; Bandow, S.; lijima, S. Effect of Fullerene Encapsulation on Radial Vibrational Breathing-Mode Frequencies of Single-Wall Carbon Nanotubes. Phys. Rev. Lett. 2009, 103, 027403.

(52) Farhat, H.; Sasaki, K.; Kalbac, M.; Hofmann, M.; Saito, R.; Dresselhaus, M. S.; Kong, J. Softening of the Radial Breathing Mode in Metallic Carbon Nanotubes. Phys. Rev. Lett. 2009, 102, 126804.

(53) Bendiab, N.; Anglaret, E.; Bantignies, J. L.; Zahab, A.; Sauvajol, J. L.; Petit, P.; Mathis, C.; Lefrant, S. Stoichiometry Dependence of the Raman Spectrum of Alkali-Doped Single-Wall Carbon Nanotubes. Phys. Rev. B 2001, 64, 245424. 
(54) Bendiab, N.; Spina, L.; Zahab, A.; Poncharal, P.; Marlière, C.; Bantignies, J. L.; Anglaret, E.; Sauvajol, J. L. Combined in Situ Conductivity and Raman Studies of Rubidium Doping of Single-Wall Carbon Nanotubes. Phys. Rev. B 2001, 63, 153407.

(55) Chen, G.; Furtado, C. A.; Bandow, S.; lijima, S.; Eklund, P. C. Anomalous Contraction of the C-C Bond Length in Semiconducting Carbon Nanotubes Observed During Cs Doping. Phys. Rev. B 2005, $71,045408$.

(56) Kataura, H.; Maniwa, Y.; Abe, M.; Fujiwara, A.; Kodama, T.; Kikuchi, K.; Imahori, H.; Misaki, Y.; Suzuki, S.; Achiba, Y. Optical Properties of Fullerene and Non-Fullerene Peapods. Appl. Phys. A 2002, 74, 349-354.

TOC :

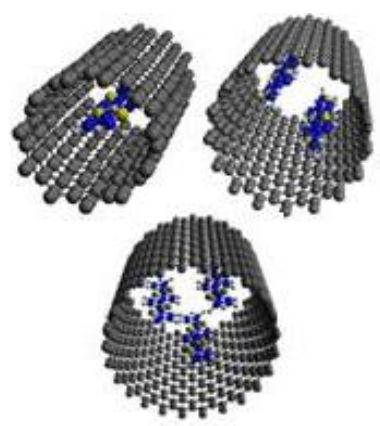

